\title{
Die Matrix der Fische
}

\author{
Von Ulrich A. Corti, Zürich
}

Eingegangen am 17 . A ugust 1948

(Mitteilung aus der Eidg. Anstalt für Wasserversorgung, Abwasserreinigung und Gewässerschutz an der Eidg. Technischen Hochschule in Zürich.)

I.

ClAUDE BERNARD hat in seinen Legons sur les phénomènes de la vie communs aux animaux et aux pégétaux $x^{\mathrm{I}}$ ) u. a. die Begriffe des «Milieu extérieur» und «Milieu intérieur» geprägt, zwei termini tecbnici, deren Wert für biotopologische Untersuchungen besonders hervorgehoben zu werden verdient.

Kombiniert man die beiden jedes singuläre Lebewesen kennzeichnenden Milieux, so gelangt man zu einer Einheit, die der Verfasser bereits an anderer Stelle ${ }^{2}$ ) als Matrix (= Existenzfeld) des Individuums bezeichnet hat.

Die Matrix irgendeines «einzelnen» Lebewesens läßt sich durch nachstehendes Schema (Abb. I) darstellen:

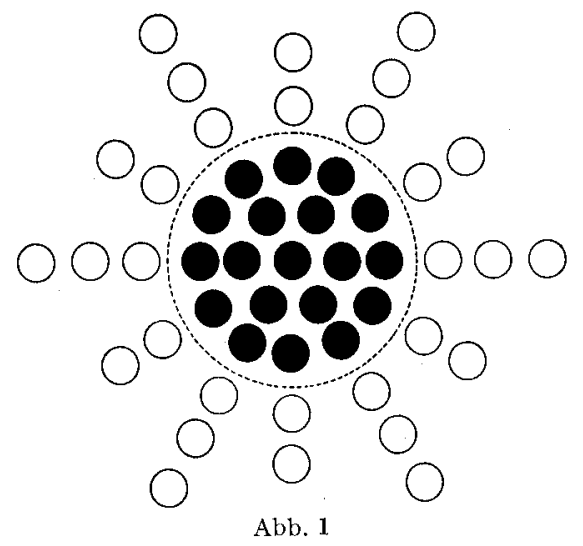

1) Bd. I, S. 26; Bd. II, S. 4f. (Paris 1879).

2) Jber, naturf. Ges. Graubünden, N. F. $80,59 \mathrm{ff}$. (1946). 
In der vorstehenden Figur mögen die durch kleine Kreise abgegrenzten Felder als Symbole für konkrete «Elemente» oder «Komponenten» sowohl des Milieu extérieur wie des Milieu intérieur eines bestimmten Lebewesens betrachtet werden. Die erwähnten «Elemente» sind also entweder konkrete Bereiche der sogenannten Außenwelt (Erdboden, Wasser, Atmosphäre usw. oder Teile solcher Naturgegebenheiten) oder Organgruppen, Einzelorgane, Zellen, Zellbausteine usw. des zu analysierenden Organismus.

Die unterbrochene Kreislinie soll die «individuelle Grenzfläche» (das Integument) des singulären Lebewesens andeuten, d. h. jene Fläche, welche die Innen- und Außenwelt des Organismus scheidet.

Die Matrix umfaßt somit die gesamte, mosaikartig strukturierte, raumzeitliche und materiellenergetische Welt des «einzelnen" Lebewesens. Letzteres steht als Subjekt nicht nur in der Mitte seiner Welt, sondern es ist identisch mit dieser Welt.

Innerhalb seiner Matrix unterliegt jedes konkrete Lebewesen in jeder Daseinsphase einer heute noch unübersehbaren Vielheit von Einflüssen (Störungen im weiteren Sinne). Außerdem übt das Lebewesen seinerseits Wirkungen auf seine Umwelt und Innenwelt aus.

Wir unterscheiden:

a)

I. Matrixelemente mit positiver Valenz, welche den Existenzformen eines konkreten Lebewesens unmittelbar oder mittelbar irgendwelche Dienste leisten. Ihre Totalität sei Dienstmatrix genannt.

2. Matrixelemente mit negativer Valenz, welche die verschiedenen Existenzformen eines Lebewesens direkt oder indirekt stören (Störungen im engeren Sinn) bzw. gefährden. Ihre Gesamtheit bezeichnen wir als Störungsmatrix.

3. Matrixelemente mit potentieller Valenz, welche sich in bezug auf die Existenzformen cines Lebewesens indifferent verhalten. Wir fassen sie im Begriff der Neutralmatrix zusammen.

b)

I. Matrixelemente eines konkreten Lebewesens, welche den Existenzformen eines oder mehrerer anderer ( $=$ fremder) Lebewesen unmittelbar oder mittelbar Dienste leisten.

2. Matrixelemente eines konkreten Lebewesens, welche die Existenzformen eines oder mehrerer anderer Lebewesen direkt oder indirekt stören bzw. gefährden.

3. Matrixelemente eines konkreten Lebewesens, welche die Existenzformen eines oder mehrerer anderer Lebewesen weder beeinträchtigen (stören) noch begünstigen.

Durch diese Gliederung wird die Matrix zu einem Wertfeld (Axiotop) geprägt. Jedes Element des Existenzfeldes weist entweder einen positiven, 
einen negativen oder einen neutralen Wert auf; jedes kann auch ambivalent sein.

In axiologischer (wertmäßiger) Prägung sieht das weiter oben mitgeteilte Schema der Matrix nun wie folgt aus (Abb. 2):

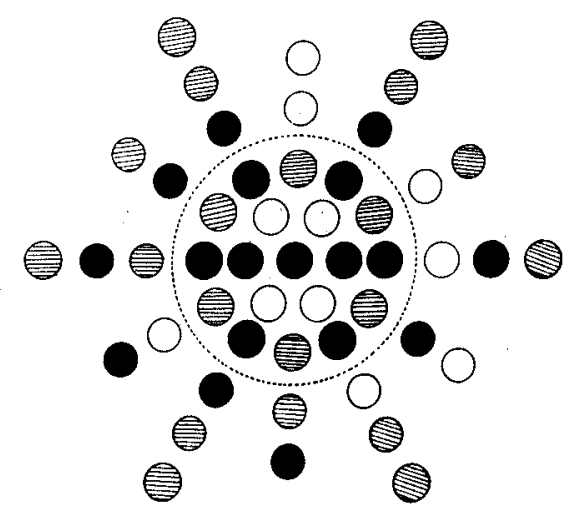

Abb. 2

Die leeren (weißen) Kreisfelder symbolisieren die Elemente der Dienstmatrix, die schwarz ausgefüllten Flächen die Störungsmatrix und die schraffierten Felder die Neutralmatrix.

Mit Hilfe dieses einfachen Schemas läßt sich die «Welt» jedes einzelnen Lebewesens systematisch untersuchen und bis in beliebige Tiefen und Feinheiten hinein abtasten.

Fügt man die bisher gewonnenen Ergebnisse biologischer Analysen, soweit letztere die Grundfrage nach den allgemein geltenden Lebensprinzipien zu beantworten versuchen, zusammen, so hat als eines dieser Prinzipien ohne Zweifel der Satz zu gelten, daß die von den Lebewesen bzw. den dieselben beherrschenden Mächten primär geleistete Arbeit in der Entfaltung und Erhaltung organischer bzw. vitaler Existenz besteht. Dieser Satz enthält implizite die grundlegende Tatsache der Gefährdung eben dieser vitalen Existenz durch Störungsfaktoren der Matrix, denen die Sicherungsfunktionen des Organismus als Dienstmatrixelemente ( $=$ Lebensmittel sensu lato) gegenüberstehen.

Vorläufig ist noch auf folgenden bemerkenswerten Umstand hinzuweisen. Das Milieu extérieur des Individuums enthält Elemente, die nicht weniger lebensnotwendig (unentbehrlich, essentiell) sind als solche des Milieu intérieur. Diese vielleicht etwas trivial klingende Feststellung be- 
sagt, daß sich die beiden Milieutypen prinzipiell wie Matrize und Patrize oder wie Positiv und Negativ ergänzen. Zwischen dem Milieu intérieur und Milieu extérieur besteht eine komplementäre Allianz oder Union derart, daß die «Innenwelt» des Individuums ohne eine adäquate Außenwelt einfach unmöglich ist.

So bildet z. B. der Sauerstoffgehalt des als Aufenthaltsmedium der Fische dienenden Wassers das lebensnotwendige Komplement der Atmungsorgane (Kiemen, Blut). Das Verdauungs-, Assimilations- und Exkretionssystem der Fische setzt grundsätzlich die Zufuhr von exogener Nahrung und von Wasser sowie die Möglichkeit zur Abgabe von Exkrementen, das Stationieren bzw. Dislozieren unserer Süßwasserbewohner das Vorhandensein entsprechender Organe (wie Flossen, Schwimmblase, Muskelsystem usw.) voraus.

Wenn nun gewisse Elemente des Milieu extérieur kategorial als integrierende Wesenheiten des Milieu intérieur zu taxieren sind, so wird dadurch die prinzipielle Undefinierbarkeit des Begriffes der Individualität in raumzeitlicher und materiellenergetischer Hinsicht grell beleuchtet.

Es ist mit andern Worten die Annahme, jedes Lebewesen sei durch sein Integument "definiert», durchaus willkürlich. Sie sollte nach unserem Dafürhalten aufgegeben und durch eine wesentlich erweiterte Konzeption ersetzt werden. Hierfür eignet sich der Begriff der Matrix vorzüglich als Ausgangsbasis.

In der Wirklichkeit erweist sich die integumentale Grenzfläche des Lebewesens in ihrem materiellen Feinbau als um so mannigfaltiger, sozusagen siebartig durchbrochen, je weiter die Analyse vorgetrieben wird. Damit entgleitet auch jede Möglichkeit ihrer absolut scharfen, d. h. eindeutigen Definition, z. B. mit Hilfe mathematischer Formulierungen. Im übrigen offenbart sich auch hier eindrücklich das Wesen der Individualität im Sinne unteilbarer Existenz.

Gegenüber solchen Schwierigkeiten empfiehlt es sich, jede individuelle Matrix als ein netz- oder mosaikartiges System von Relationen zwischen Elementen der Umwelt und der Innenwelt, als ein stark differenziertes Feld verschiedenartiger Naturgegebenheiten aufzufassen, dessen Auflösung zwangsläufig nur unter teilweiser oder gänzlicher Zerstörung des Lebewesens möglich ist.

Der «Constitution interne» des Lebewesens ist eine «Constitution externe» konjugiert zugeordnet, wobei unter «Konstitution» eine von Fall zu Fall verschiedene Kombination (Ordnung, System, Gefüge, Hierarchie) 
von Lebensmitteln sensu lato (d. h. von Dienstmatrixelementen) zu verstehen ist.

Das einzelne Lebewesen ist darnach, um hier einen trefflichen Ausdruck von $\mathrm{H}$. WeYL anzuwenden, in Wirklichkeit eine Welt, die nach innen wie nach außen offen ist. Mit dieser Einsicht wird der Begriff des Individuums im traditionellen Sinne nur noch mit besonderen Vorbehalten als Operator verwendet werden können.

.2 .

Zur Analyse der Grundfunktionen der Lebewesen bedienen wir uns wiederum eines Schemas (Abb. 3) und erläutern dasselbe an konkreten, dem Reiche der Süßwasserfische entnommenen Beispielen.

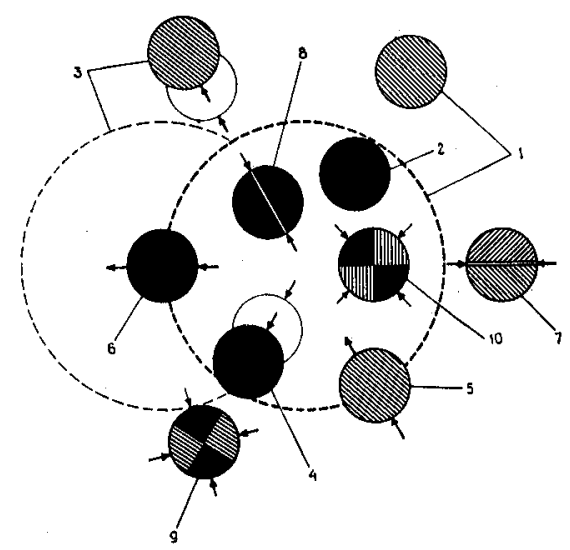

Abb. 3

Die durch kleine Kreise abgegrenzten Felder symbolisieren auch hier Elemente der Matrix, also der Außen- und Innenwelt eines Lebewesens.

Es zeigt sich, daß sich sämtliche Funktionen jedes Lebewesens, z. B. eines Flußbarsches, Perca fluviatilis L., wie überhaupt alle sich in der individuellen Matrix abspielenden Ereignisse auf folgende allgemeine Geschehnisse zurückführen lassen:

r. Relatives Verharren (Stationieren) beliebiger Matrixelemente (inbegriffen das sogenannte Individuum, z. B. eines Aals, Anguilla pulgaris L.) an einem bestimmten Ort des Milieu extérieur.

2. Relatives Verharren beliebiger Matrixelemente (z. B. der Leber) im Milieu intérieur. 
3. Relative Dislokation beliebiger Matrixelemente (inbegriffen das sogenannte Individuum, z.B. eines Hechtes, Esox lucius L.; oder von Beuteobjekten) im Milieu extérieur.

4. Relative Dislokation beliebiger Matrixelemente (z. B. von Blutkörperchen) im Milieu intérieur.

5. Aufnahme ("Verinnerung») von Matrixelementen (z. B. von Sauerstoff) aus dem Milieu extérieur durch das Milieu intérieur.

6. Abgabe ("Veräußerung») von Matrixelementen (z. B. von Exkrementen) durch das Milieu intérieur an das Milieu extérieur.

7. Trennung von Matrixelementen des Milieu extérieur (z. B. das Auseinandertreiben eines geschlossenen Kleinfischschwarmes durch jagende Raubfische).

8. Trennung von Matrixelementen im Milieu intérieur (z. B. Zellteilung).

9. Kombination von Matrixelementen im Milieu extérieur (z. B. Zusammenfügung von verschiedenen Baustoffen zu einem Nest durch den Stichling, Gasterosteus aculeatus L.).

Io. Kombination von Matrixelementen im Milieu intérieur (z. B. Assimilation von Nahrungsmitteln).

Diese in der Abb. 3 bildlich dargestellten zehn Grundtypen von Vorgängen bzw. Ereignissen umfassen wohl alle möglichen Hauptvarianten von Geschehnissen in der Matrix eines Lebewesens, soweit sie naturwissenschaftlicher Analyse zugänglich sind.

Man erkennt ohne weiteres, daß es sich dabei durchwegs um die Separation oder Kombination von Matrixelementen handelt. Erfahrungsgemäß erfolgt die Lagerung, Verlagerung, Trennung oder Verbindung von Matrixelementen in der Biosphäre nach gewissen, z. T. bekannten, z. T. unbekannten Normen (Prinzipien). Die Feststellung dieser Normen ist eine der vornehmsten Aufgaben der Naturforschung.

Unter Berücksichtigung des im Zusammenhang mit den Abb. I bis 3 Gesagten, gelangt man zu nachstehender Darstellung des integralen Komplexes der vitalen Grundfunktionen.

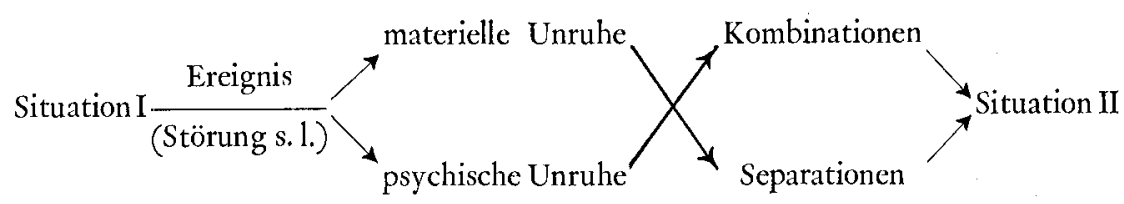

Für die aus den Unruhen resultierenden Aktionen bzw. Ereignisse, die integral aus Kombinationen und Separationen (siehe Abb. 3) bestehen, ergibt sich folgendes biologisches Schema: 


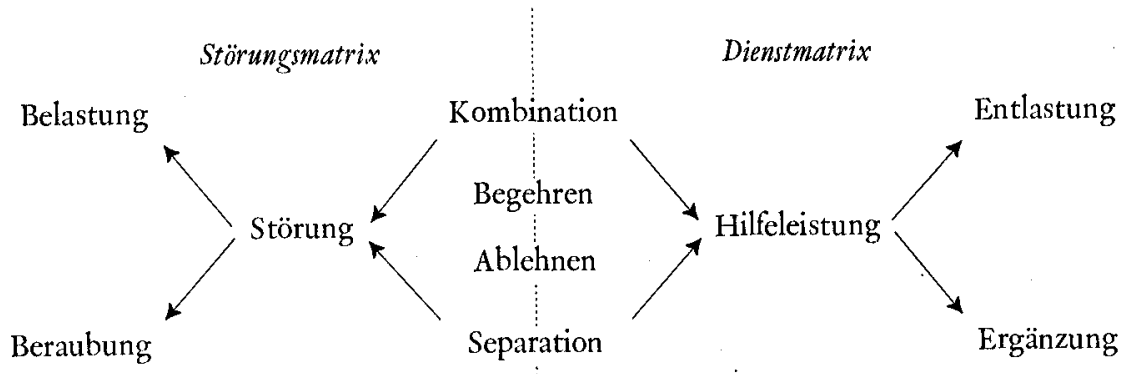

Durch die vertikale, unterbrochene Linie wird der gesamte Komplex der vitalen Funktionen in die Störungsmatrix (linke Hälfte des Schemas) und die Dienstmatrix (rechte Hälfte des Schemas) des Individuums geteilt.

Unter Allotrioaxie ist der biologische Wert eines konkreten Individuums (auch von Komponenten und Funktionen desselben) in bezug auf sein Milieu extérieur, unter Autoaxie der Wert der gesamten Matrix in bezug auf die vitale Existenz des Individuums als Subjekt zu verstehen.

Innerhalb der Störungsmatrix ist im allotrioaxischen Sektor die Gesamtheit der Störungen zu untersuchen, die ein Subjekt in seinem Milieu extérieur auslöst bzw. verursacht. Diese Störungen können eine Belastung anderer vitaler Existenzen mit Bürden irgendwelcher Art darstellen oder auf dem Entzug von Lebens- bzw. Existenzmitteln beruhen.

Im autoaxischen Sektor der Störungsmatrix unterliegt das Subjekt selbst der Störung durch Belastung oder Beraubung. Hier muß im Interesse der Erhaltung und Entfaltung von "Individuum und Art» die Abwehr (Ablehnung) des Subjekts einsetzen.

Das allotrioaxische «Feld» der Dienstmatrix des Subjekts umfaßt jene Gruppe von Funktionen, die, sei es durch Ergänzungs-, sei es durch Entlastungsoperationen, Lebewesen im Milieu extérieur irgendwelche Dienste leisten. Im autoaxischen «Feld» vollziehen sich dagegen diejenigen Operationen, die dem Subjekt durch Entlastungs- oder Ergänzungsleistungen dienen. Solche Leistungen werden vom Subjekt, seinem Wesen entsprechend, begehrt.

Die elementaren Grundfunktionen des Störens, Dienens, Ablehnens und Begehrens haben ihren Ursprung im Subjekt. Sie werden offenbar ausgelöst auf Grund von Wahrnehmungen ( Sensationen»), die das Individuum im Milieu extérieur oder Milieu intérieur macht. Jede Wahrnehmung ist 
mit einer Differenzierungsoperation verkettet, derart, daß auf Grund der Ergebnisse der Wahrnehmung und Differenzierung (als Entscheidung) des Wahrgenommenen eine Wertung (Taxierung, Beurteilung) erfolgt, die nun zum auslösenden Moment der vorerwähnten Grundfunktionen wird. Das Ergebnis ist die Scheidung der Matrixelemente nach positiven, negativen und neutralen Valenzen (Abb. 2).

Es bietet eminentes Interesse, die "Wertposition» (Wertigkeit im biologischen Sinne) und hernach die hierarchische Struktur der Wertmatrix (des Axiotops $=$ Wertfeld) möglichst erschöpfend kennenzulernen. Diese Wertmatrix widerspiegelt mutmaßlich unmittelbar das eigentliche Wesen des Subjekts.

Die mit der vorliegenden Einführung in die allgemeine Problematik vitaler Existenz eröffnete Studienserie soll am konkreten Beispiel der Fischwelt analysiert werden.

\section{SUMMARY}

In the first of a series of publications on the conditions of living of fish, there is demonstrated that the "milieu extérieur" and the "milieu intérieur" of all creature are strictly correlated and that the living being can be interpreted as a combination of indispensable factors of existence. It is possible to reduce all vital basic functions to a limited number of combinations or separations of material unities. The ro elementary operations for combining or separating those unities are tabulated in a schematic form. 